
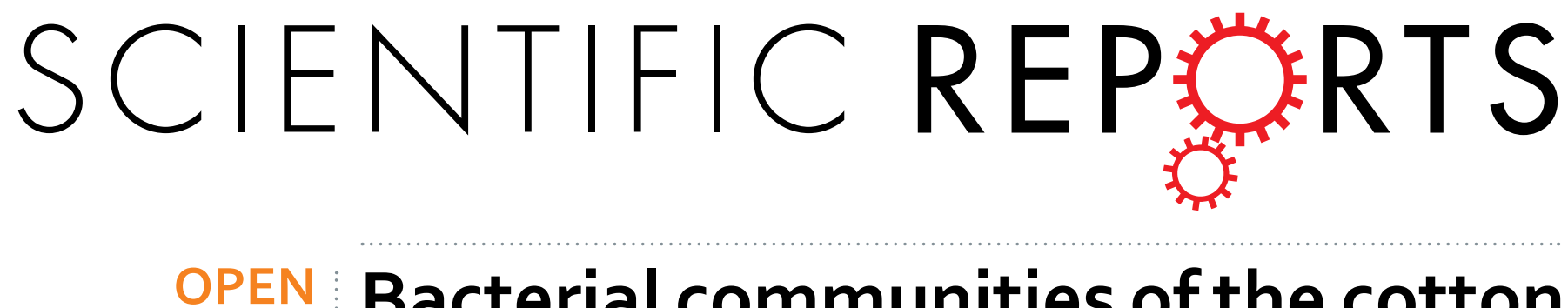

\title{
Bacterial communities of the cotton aphid Aphis gossypii associated with $B t$ cotton in northern China
}

Received: 09 December 2015

Accepted: 16 February 2016

Published: 15 April 2016

\author{
Yao Zhao ${ }^{1,2}$, Shuai Zhang ${ }^{1}$, Jun-Yu Luo ${ }^{1}$, Chun-Yi Wang ${ }^{1}$, Li-Min Lv ${ }^{1} \&$ Jin-Jie Cui ${ }^{1}$
}

Aphids are infected with a wide variety of endosymbionts that can confer ecologically relevant traits. However, the bacterial communities of most aphid species are still poorly characterized. This study investigated the bacterial diversity of the cotton aphid Aphis gossypii associated with Bt cotton in northern China by targeting the $\mathrm{V} 4$ region of the $16 \mathrm{~S}$ rDNA using the Illumina MiSeq platform. Our sequencing data revealed that bacterial communities of $A$. gossypii were generally dominated by the primary symbiont Buchnera, together with the facultative symbionts Arsenophonus and Hamiltonella. To our knowledge, this is the first report documenting the facultative symbiont Hamiltonella in A. gossypii. Moreover, the bacterial community structure was similar within aphids from the same province, but distinct among those from different provinces. The taxonomic diversity of the bacterial community is greater in Hebei Province compared with in samples from Henan and Shandong Provinces. The selection pressure exerted by the different geographical locations could explain the differences found among the various provinces. These findings broaden our understanding of the interactions among aphids, endosymbionts and their environments, and provide clues to develop potential biocontrol techniques against this cotton aphid.

Aphids engage in symbiotic associations with a diverse assemblage of heritable bacteria. Aphid-associated bacterial community can vary with the sex and genotype of the insect host, and with environmental conditions, including temperature and $\operatorname{diet}^{1-4}$. Bacteria that are restricted to specialized insect cells and obligately vertically transmitted are known as primary symbionts ${ }^{5}$. Almost all aphids require the primary symbiont, Buchnera aphidicola, which provides nutrients not obtained in sufficient quantities from plant phloem ${ }^{4,6}$. Many phloem-sap-feeding insects also contain one to several other bacteria, called secondary or facultative symbionts, which may be localized to the bacteriocytes, other insect cells or the body cavity, and are capable of both vertical and horizontal transmission ${ }^{7}$.

While some aphids carry only the obligate symbiont B. aphidicola, most pea aphids, Acyrthosiphon pisum, are additionally infected with one or more facultative symbionts ${ }^{8,9}$. These symbionts confer various phenotypes to $A$. pisum, including defence against parasitism, protection against fungal pathogens, tolerance to heat stress, plant usage and reproductive manipulation ${ }^{10-14}$. Documenting the presence of facultative symbionts and identifying their effects on hosts can have important implications for the management of pest species.

A. pisum has been used as a model insect to study several questions related to the diversity and interactions of symbiotic bacteria with aphids ${ }^{4}$, but little information is available on the bacteria associated with other aphid species. High-throughput DNA sequencing approaches provide a new way to characterize bacterial communities, and this approach permits the investigation of ecological questions that could not be addressed using traditional methods $^{15,16}$

The cotton aphid, Aphis gossypii, has a worldwide distribution and causes damage to numerous economically important crops $^{17}$. However, the bacterial communities harboured by A. gossypii are still poorly characterised. In the present study, we used the Illumina MiSeq platform targeting the V4 region of the $16 \mathrm{~S}$ rDNA to determine (1) whether the bacterial communities in A. gossypii associated with Bt cotton were dominated by the primary and facultative symbionts, and (2) whether the diversity of the bacterial communities differed among multiple field populations of A. gossypii in northern China.

${ }^{1}$ State Key Laboratory of Cotton Biology, Institute of Cotton Research of CAAS, Anyang 455000, China. ${ }^{2}$ Hubei Insect Resources Utilization and Sustainable Pest Management Key Laboratory, Huazhong Agricultural University, Wuhan 430070, China. Correspondence and requests for materials should be addressed to J.-J.C. (email: cuijinjie@126.com) 


\begin{tabular}{|l|c|c|c|c|c|c|c|c|}
\hline Province & Sample & $\begin{array}{c}\text { No. } \\
\text { reads }\end{array}$ & $\begin{array}{c}\text { No. } \\
\text { OTU* }\end{array}$ & Ace & Chaol & Shannon & Simpson & Coverage \\
\hline Henan & Zhoukou(Zk) & 14,741 & 215 & 208.26 & 203.18 & 1.58 & 0.33 & 1.00 \\
\hline Henan & Xuchang(Xc) & 58,353 & 223 & 243.41 & 195.81 & 1.13 & 0.26 & 1.00 \\
\hline Henan & Shangqiu(Sq) & 11,241 & 107 & 124.70 & 119.33 & 0.88 & 0.19 & 1.00 \\
\hline Hebei & Cangzhou(Cz) & 59,694 & 1034 & 1045.13 & 964.19 & 3.24 & 0.55 & 0.97 \\
\hline Hebei & Hengshui(Hs) & 38,368 & 874 & 818.90 & 765.79 & 2.27 & 0.39 & 0.98 \\
\hline Hebei & Handan(Hd) & 45,373 & 993 & 1093.36 & 975.18 & 3.02 & 0.49 & 0.97 \\
\hline Shandong & Jining(Jn) & 44,998 & 248 & 240.37 & 211.67 & 0.68 & 0.13 & 0.99 \\
\hline Shandong & Bingzhou(Bz) & 12,737 & 140 & 143.92 & 145.05 & 1.87 & 0.47 & 1.00 \\
\hline Shandong & Heze(Hz) & 28,603 & 163 & 171.84 & 164.56 & 0.65 & 0.14 & 1.00 \\
\hline Shandong & Dezhou(Dz) & 24,088 & 490 & 576.51 & 505.66 & 1.93 & 0.39 & 0.98 \\
\hline
\end{tabular}

Table 1. Sequencing analysis of $16 \mathrm{~S}$ rRNA gene amplicons of $A$. gossypii with diversity indices. ${ }^{\star}$ Operational taxonomic units (OTUs) were defined with pairwise $97 \%$ sequence identity.

\begin{tabular}{|c|c|c|c|c|c|c|c|c|c|c|c|}
\hline \multirow[b]{2}{*}{ Province } & \multirow[b]{2}{*}{ Site } & \multirow[b]{2}{*}{ Latitude/Longitude } & \multicolumn{9}{|c|}{ Relative abundances (\%) } \\
\hline & & & $A$ & $B$ & $H$ & $\operatorname{Re}$ & $R i$ & $\mathrm{Se}$ & $S p$ & $W$ & Other \\
\hline Henan & Zhoukou(Zk) & $33^{\circ} 45^{\prime} \mathrm{N}, 114^{\circ} 27^{\prime} \mathrm{E}$ & 2.70 & 83.06 & 0.14 & 0 & 0 & 0 & 0 & 0 & 14.10 \\
\hline Henan & Xuchang(Xc) & $33^{\circ} 50^{\prime} \mathrm{N}, 114^{\circ} 14^{\prime} \mathrm{E}$ & 4.74 & 87.93 & 0.11 & 0 & 0 & 0 & 0 & 0 & 7.22 \\
\hline Henan & Shangqiu(Sq) & $34^{\circ} 31^{\prime} \mathrm{N}, 115^{\circ} 42^{\prime} \mathrm{E}$ & 2.80 & 91.68 & 0.78 & 0 & 0 & 0 & 0 & 0.01 & 4.73 \\
\hline Hebei & Cangzhou $(\mathrm{Cz})$ & $37^{\circ} 58^{\prime} \mathrm{N}, 116^{\circ} 49^{\prime} \mathrm{E}$ & 3.23 & 71.51 & 0.17 & 0 & 0 & 0 & 0 & 0.01 & 25.08 \\
\hline Hebei & Hengshui(Hs) & $37^{\circ} 31^{\prime} \mathrm{N}, 115^{\circ} 39^{\prime} \mathrm{E}$ & 0.31 & 84.73 & 0.20 & 0 & 0 & 0 & 0 & 0 & 14.76 \\
\hline Hebei & Handan(Hd) & $36^{\circ} 47^{\prime} \mathrm{N}, 115^{\circ} 00^{\prime} \mathrm{E}$ & 2.30 & 76.40 & 0.18 & 0 & 0 & 0 & 0 & 0 & 21.12 \\
\hline Shandong & Jining(Jn) & $35^{\circ} 01^{\prime} \mathrm{N}, 116^{\circ} 18^{\prime} \mathrm{E}$ & 1.40 & 95.18 & 0.10 & 0 & 0 & 0 & 0 & 0 & 3.32 \\
\hline Shandong & Bingzhou $(\mathrm{Bz})$ & $37^{\circ} 44^{\prime} \mathrm{N}, 117^{\circ} 39^{\prime} \mathrm{E}$ & 2.24 & 73.57 & 0.68 & 0 & 0 & 0 & 0 & 0.01 & 23.50 \\
\hline Shandong & $\operatorname{Heze}(\mathrm{Hz})$ & $34^{\circ} 47^{\prime} \mathrm{N}, 115^{\circ} 59^{\prime} \mathrm{E}$ & 2.51 & 94.89 & 0.10 & 0 & 0 & 0 & 0 & 0 & 2.50 \\
\hline Shandong & Dezhou(Dz) & $37^{\circ} 00^{\prime} \mathrm{N}, 116^{\circ} 00^{\prime} \mathrm{E}$ & 0.99 & 84.09 & 7.07 & 0 & 0 & 0 & 0 & 0.19 & 7.66 \\
\hline
\end{tabular}

Table 2. Distribution and relative abundance of bacterial symbionts in $\mathbf{A}$. gossypii. ${ }^{a}$ Facultative symbiont abbreviations: A, Arsenophonus; B, Buchnera; H, Hamiltonella; Re, Regiella; Ri, Rickettsia; Se, Serratia; Sp, Spiroplasma; W, Wolbachia.

\section{Results}

Sequencing data. The Illumina MiSeq sequencing of the $16 \mathrm{~S}$ rRNA gene amplicons from the field samples of the cotton aphid A. gossypii yielded 11,241-59,694 raw reads per sample (Table 1). After quality filtering and the removal of chimeric sequences, 10,710-57,034 reads per sample remained. The reads for the aphid samples could be assigned to 1358 OTUs, at $97 \%$ sequence identity. The rarefaction curve for every sample tended to saturation (Fig. S1), and the value of Good's coverage of sequencing data in all samples was above $97 \%$ at the 0.03 dissimilarity cut-off (Table 1). These results indicated that our sequencing results captured most of the bacterial diversity associated with A. gossypii.

Bacterial diversity in A. gossypii. Bacterial communities in the aphid samples were dominated by the phylum Proteobacteria, with a relative abundance of $95.56 \%$ (average values across all samples) (Fig. S2). In addition, Proteobacteria had four sub-phyla that dominated the aphid-associated communities (average abundance values across all samples): Gammaproteobacteria (90.47\%), Alphaproteobacteria (2.57\%), Deltaproteobacteria (1.75\%) and Betaproteobacteria (0.68\%) (Fig. S3).

At the family level, Enterobacteriaceae was the most dominant, with a relative abundance above 75\% (Fig. S4). The relative abundances at the genus level are presented in Table 2. All aphid samples bore the primary symbiont Buchnera and two facultative symbionts, Arsenophonus and Hamiltonella. Buchnera dominated the bacterial communities in the cotton aphid A. gossypii. The least relative abundance of Buchnera was from the sample Cz, at $71.55 \%$, and the highest was from the sample Jn, at $95.21 \%$. The genera Arsenophonus was well-represented with relative abundances of $0.31 \%-4.74 \%$. The relative abundance of Hamiltonella in all of the samples was less than $1 \%$, except sample Dz. Furthermore, only samples from Cz, Sq, Bz and Dz bore the facultative symbiont Wolbachia, with relative abundances of less than $0.2 \%$. Other facultative symbionts, such as Regiella, Rickettsia, Serratia and Spiroplasma, were not found in any of the collected samples (Table 2).

Comparisons of bacterial communities from different provinces. The samples from Hebei Province were richer, having a higher number of operational taxonomic units (OTUs) than the samples from Henan and Shandong Provinces (Table 1). At the family level, the samples from Hebei generally also had more OTUs than those from Henan and Shandong, when the relative abundances of the top 35 OTUs were compared (Fig. 1). Additionally, the samples from Hebei had generally higher Ace and Chaol richness estimates compared with the samples from Henan and Shandong (Table 1). Shannon and Simpson diversity indices also suggested that 


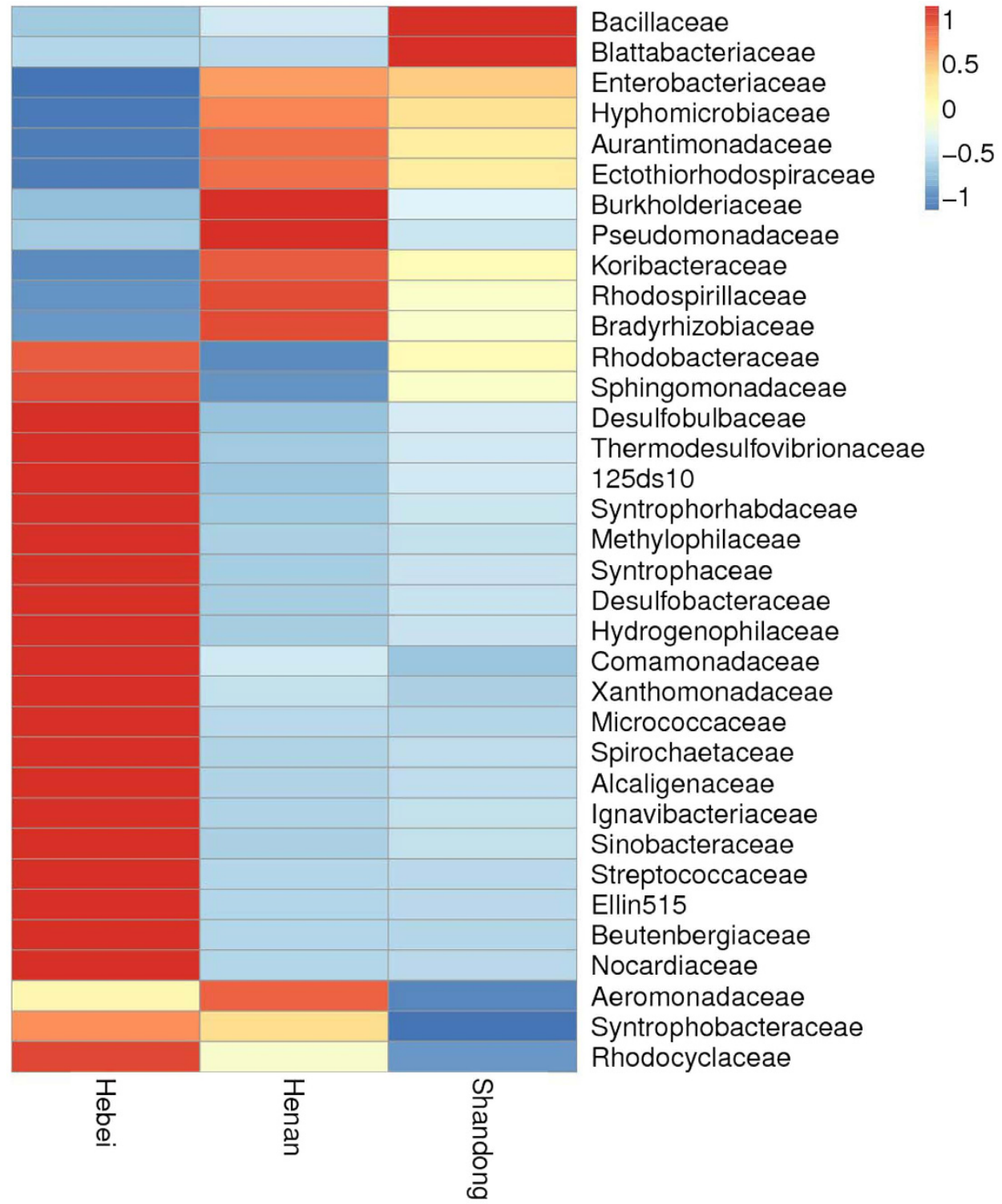

Figure 1. Heat maps showing the relative abundance and distribution of $A$. gossypii bacterial family in three provinces of northern China. The color code indicates relative abundance, ranging from blue (low abundance) to yellow to red (high abundance).

the taxonomic diversity of the bacterial community is higher in Hebei than in Henan and Shandong (Table 1). Principal coordinate analysis ( $\mathrm{PCoA}$ ) showed a distinct clustering among the individual samples, and the samples from same province tended to cluster together (Fig. 2).

\section{Discussion}

Our sequencing data revealed that the bacterial communities in A. gossypii were generally dominated by the universally present primary symbiont Buchnera. Two facultative symbionts, Arsenophonus and Hamiltonella, were also found in all of the aphid samples. To our knowledge, this is the first report finding the facultative symbiont Hamiltonella in A. gossypii. Moreover, the bacterial community structure was similar within the same province, but distinct among different provinces (Fig. 2). Our results suggest that the bacterial diversity of A. gossypii is related to the geographical location.

To utilize phloem sap as their sole dietary component, most aphids are critically dependent on symbiosis with the bacteria B. aphidicola ${ }^{6}$. Buchnera was also the predominant genus found in the bacterial communities of our aphid samples. Previous studies have shown that facultative symbionts from five genera can infect A. gossypii: Arsenophonus, Regiella, Rickettsia, Serratia and Wolbachia ${ }^{18-24}$. Among these, Arsenophonus was found in all of our samples, Wolbachia was only detected in some of our samples, and they both had low relative abundances. The other three symbionts were not detected in our sequencing data. Brady et al. summarized the infection rates 

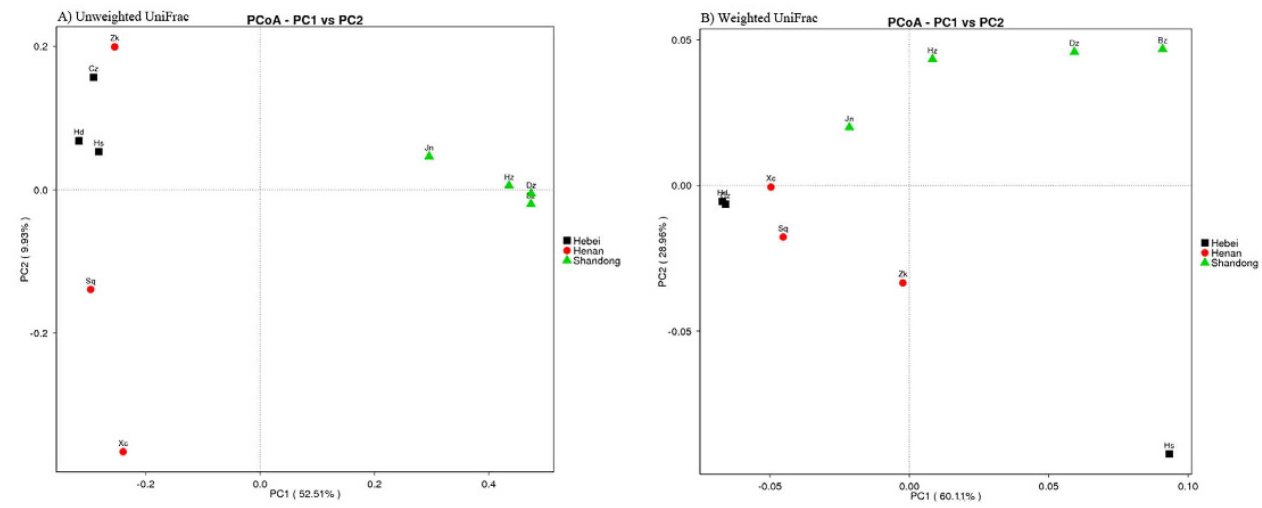

Figure 2. Comparison of bacterial community structures in A. gossypii samples in three provinces of northern China. Unweighted and weighted UniFrac metrics were used to determine pairwise distances between all samples.

of Regiella, Rickettsia and Serratia in A. gossypii, and each of them was only $1 \%{ }^{25}$. This may explain why these three facultative symbionts were not found in our results.

Arsenophonus is widespread in numerous insect species ${ }^{26}$. It can manipulate the reproduction of various parasitoid wasps by distorting the progeny sex ratio towards the production of females through male killing ${ }^{26,27}$. Arsenophonus may also behave as an obligate mutualist in hematophagous insects ${ }^{28}$, or as a facultative mutualist, protecting against parasitoid attacks in psyllids ${ }^{29}$. Jousselin et al. identified aphids as harbouring an important diversity of Arsenophonus strains, and the incidence was especially high in the Aphis genus ${ }^{24}$. Jousselin et al. also speculate that plant mediation and parasitism might be involved in the dispersal of Arsenophonus ${ }^{24}$. Moreover, Arsenophonus was reported to be involved in host plant specialization in the polyphagous aphid, Aphis craccivora $^{30}$. In recent studies, Arsenophonus did not defend its aphid host Aphis glycines against major parasitoids and fungal natural enemies ${ }^{31}$, but provided a general benefit to $A$. glycines $^{32}$.

Wolbachia is typically associated with manipulating the reproduction of several arthropod hosts, and it can rapidly reach a high frequency in host species as a consequence ${ }^{14,33}$. Additionally, Wolbachia has been implicated in providing a defence against viruses in other insects ${ }^{34}$, and it also can provide vitamin B to the host insect ${ }^{35}$. In our study, Wolbachia was only found in some of the aphid samples and their relative abundance was extremely low. Similarly, Liu et al. found a low titre of Wolbachia in A. glycines ${ }^{36}$. The detection of Wolbachia in aphids has some difficulties, which likely has resulted in it being under reported. One major difficulty is the current PCR protocols for the detection of Wolbachia were far from optimal ${ }^{18}$. In addition, the development of efficient Wolbachia detection was hindered by the presence of low titre infections and multiple infections ${ }^{37,38}$. Wolbachia density may be affected by co-infection with other Wolbachia strains or other vertically transmitted symbionts, as well as by host genotype $\mathrm{e}^{39,40}$. Another difficulty in detection of Wolbachia is horizontal transfer of Wolbachia genes to host genomes $^{41,42}$, which further complicates Wolbachia detection.

Surprisingly, Hamiltonella, which has not been reported in A. gossypii, was found in our data. Hamiltonella is known to protect aphids against parasitism. Oliver et al. found that Hamiltonella defensa reduced the rate of successful parasitism by Aphidius ervi by killing developing wasp larvae ${ }^{43}$. Multiple strains of $H$. defensa were examined in A. pisum and all of them conferred a partial protection against attack by A. ervi, indicating that $H$. defensa generally provides protection against this wasp ${ }^{10}$. In addition, parasitized A. pisum containing $H$. defensa produced significantly more offspring than parasitized uninfected aphids, indicating that $A$. pisum benefited from the $H$. defens $a$ infection when under attack by parasitoids ${ }^{44}$. H. defensa being present in all of our samples may also suggest the importance of its role in protecting $A$. gossypii against parasitism in the field, and both vertical and horizontal transmission may act as drivers of Hamiltonella dispersal.

Many other bacterial taxa were also detected in some of the aphid samples, and their relative abundances were extremely low. For example, Stenotrophomonas, Brevundimonas and Burkholderia, which are commonly detected in environmental samples, were also found in our aphid samples ${ }^{22,23}$. Burkholderia is present in the environment, associated with insects and, in some instances, clearly acts as a mutualist ${ }^{45}$. Many of these bacteria could be contaminants. Additionally, our aphid samples from 10 different field locations could encounter different environmental factors. Although heritability cannot be ruled out, it is more likely that these bacteria engage in opportunistic associations with aphids (perhaps as gut associates or pathogens) or that they represent contaminants from soil, plants or human handling.

Geographical location and environmental factors may account for the different bacterial community structures found in aphids from different provinces. Natural populations of aphids may experience selection pressures from various management practices, natural enemies (pathogens, predators and parasitoids) and environmental conditions that could alter the composition, as well as the frequency, of associated bacteria ${ }^{46}$. In the PCoA, samples from the same province tended to cluster together (Fig. 2), suggesting that the samples might have experienced similar selection pressures.

In our results, the samples from Hebei Province had a higher number of OTUs, higher Ace and Chaol richness estimates, and higher Shannon and Simpson diversity indices, compared with the samples from Henan and Shandong Provinces (Table 1). This may be because Hebei Province has a relatively higher latitude and may 


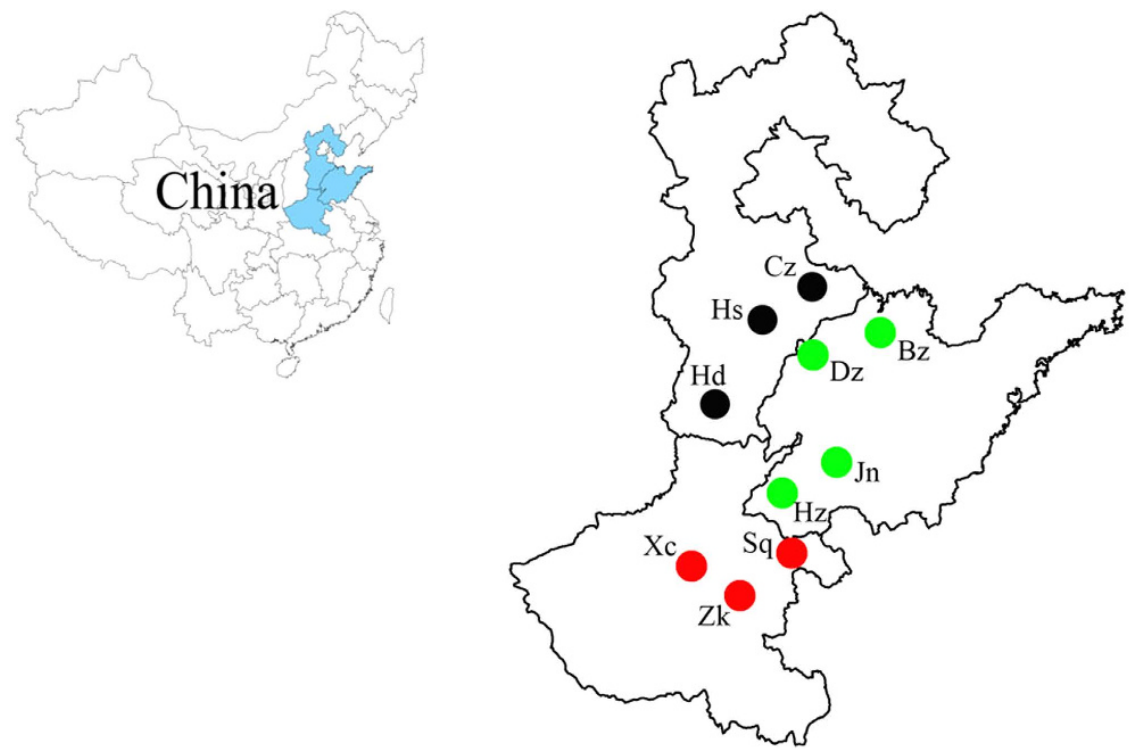

$200 \mathrm{~km}$

Figure 3. Sampling locations of $\boldsymbol{A}$. gossypii in three provinces of northern China. Henan Province: Zhoukou(Zk), Xuchang(Xc), Shangqiu(Sq). Hebei Province: Cangzhou(Cz), Hengshui(Hs), Handan(Hd). Shandong Province: Jining(Jn), Bingzhou(Bz), Heze(Hz), Dezhou(Dz). The map is created with ArcGIS 10.2 (http://www.arcgis.com/features/).

encounter different climactic variables, including temperature and precipitation. A geographical-based variation in infection frequencies has been reported for some facultative symbionts. In the pea aphid, A. pisum, symbiont prevalence was found to correlate with climactic variables ${ }^{47}$. Bacteria from the whitefly Bemisia tabaci differ in frequency based on the host plants and geographical locations ${ }^{48}$. Moreover, geographical variation in Arsenophonus symbiont prevalence was reported in the psyllid Glycaspis brimblecombei ${ }^{29}$. These studies suggest that environmental factors may act as important drivers of natural symbiont dynamics.

Here, we used the deep Illumina MiSeq sequencing of 16S rDNA genes, to analyse the bacterial communities of the cotton aphid A. gossypii associated with Bt cotton in northern China. Our sequencing data revealed that the bacterial communities of A. gossypii were dominated by the primary symbiont Buchnera, together with facultative symbionts that varied in incidence among the aphid samples. To our knowledge, this is the first report documenting the facultative symbiont Hamiltonella in A. gossypii. Selective pressures exerted by the geographical location could explain why the bacterial community structure was similar within the same province, but distinct among different provinces. These findings increase our understanding of the intricate symbiotic relationships between bacteria and A. gossypii. Further studies will be focused on identifying the functions of the representative bacterial species and determining whether these species could play important roles in the future as biocontrol agents.

\section{Methods}

Insect sampling and DNA extraction. Apterous adults of Aphis gossypii were collected from 10 field populations in northern China during August 2014 (Table 2 and Fig. 3). All of the sampling sites were planted with CrylAc cotton. The aphids were immediately immersed in $90 \%$ ethanol and frozen at $-80^{\circ} \mathrm{C}$ upon return to the laboratory.

Prior to DNA extractions, aphid samples, each comprising 20 adult aphids, were washed for $5 \mathrm{~min}$ in $70 \%$ ethanol and rinsed three times with sterile water to remove surface contaminants. Then, samples were hand-homogenized in extraction buffer $\left(20 \mathrm{mM}\right.$ Tris- $\mathrm{HCl} \mathrm{pH} 8.0,2 \mathrm{mM}$ sodium EDTA, $1.2 \%$ Triton ${ }^{\circledR} \mathrm{X}-100$ containing $20 \mathrm{mg}$ lysozyme $\mathrm{ml}^{-1}$ ). The homogenates were incubated at $37^{\circ} \mathrm{C}$ for $40 \mathrm{~min}$ to achieve DNA extraction from both Gram-positive and Gram-negative bacteria. The DNA in the samples was then extracted using the TIANamp Genomic DNA Kit (TIANGEN Biotech (Beijing) LTD., China) following the manufacturer's instructions. The quantity and quality of the DNA were measured with a NanoDrop 2000c spectrophotometer (Thermo Scientific, USA).

PCR amplification, library preparation and sequencing. DNA was amplified using the 515f/806r primer set (515f: 5' -GTG CCA GCM GCC GCG GTA A-3', 806r: 5' -XXX XXX GGA CTA CHV GGG TWT CTA AT- $3^{\prime}$ ), which targets the V4 region of the bacterial $16 \mathrm{~S}$ rDNA, with the reverse primer containing a 6-bp error-correcting barcode unique to each sample. PCR amplifications were carried out in a $30 \mu \mathrm{l}$ mixture that included $15 \mu \mathrm{l}$ of Phusion High-Fidelity PCR Master Mix (New England Biolabs, UK), $0.2 \mu \mathrm{M}$ of forward and reverse primers, $10 \mathrm{ng}$ of template DNA and nuclease-free water up to $30 \mu \mathrm{l}$. The PCR conditions were $98^{\circ} \mathrm{C}$ for $1 \mathrm{~min}(1 \mathrm{cycle}), 98^{\circ} \mathrm{C}$ for $10 \mathrm{~s}, 50^{\circ} \mathrm{C}$ for $30 \mathrm{~s}$ and $72^{\circ} \mathrm{C}$ for $60 \mathrm{~s}(30 \mathrm{cycles})$, followed by $72^{\circ} \mathrm{C}$ for $5 \mathrm{~min}$. PCR 
products were mixed in equidensity ratios and mixture of PCR products was purified using the GeneJET Gel Extraction Kit (Thermo Scientific, USA).

Sequencing libraries were generated using a NEB Next Ultra DNA Library Prep Kit for Illumina (New England Biolabs, UK). The final quality and concentration of each library were checked using Agilent 2100 Bioanalyzer Instruments (Agilent Technologies, USA) and determined using KAPA Library Quantification Kits (Kapa Biosystems, USA). Sequencing was conducted on an Illumina MiSeq $2 \times 250$ platform at Novogene Bioinformatics Technology (Beijing, China) according to protocols described by Caporaso et al. ${ }^{49}$ and Kozich et al. ${ }^{50}$.

Bioinformatics and statistical analysis. Paired-end reads were assigned to samples based on their unique barcodes and truncated by cutting off the barcode and primer sequence. Then, the paired-end reads were merged into single, longer sequences using FLASH (Version 1.2.7 $)^{51}$. Quality filtering on the raw tags was performed under specific filtering conditions to obtain high-quality clean tags ${ }^{52}$ according to the QIIME (Version 1.7.0) ${ }^{15}$ quality controlled process. Chimeric sequences were detected and removed using the UCHIME algorithm ${ }^{53}$.

Sequence analyses were performed using Uparse (Version 7.0.1001) ${ }^{54}$. Sequences with $\geq 97 \%$ similarity were assigned to the same OTU. Representative sequences from each OTU were screened for further annotation. For each representative sequence, the GreenGene Database ${ }^{55}$ was used with the RDP classifier (Version 2.2 ) $^{56}$ to annotate taxonomic information. To study the phylogenetic relationships of different OTUs, and the differences in the dominant species of different samples, multiple sequence alignments were conducted using MUSCLE (Version 3.8.31) ${ }^{57}$.

To account for inequalities in sequence read depths among the samples, a randomly selected subset of 10,435 sequences per sample was chosen for a further bacterial community analysis. The microbial diversity was analysed using QIIME V1.7.0 and displayed with R software (Version 2.15.3) ${ }^{15}$. Alpha diversity analysis included observed species, Ace and Chaol estimators, Simpson and Shannon diversity indices and Good's estimate of coverage. A PCoA ${ }^{16}$ was performed to explore the differences in the bacterial community structures and was displayed with the WGCNA, stat and ggplot2 packages in the R software (Version 2.15.3). The sequencing data has been submitted to the NCBI database as a file under accession number SRP066541.

\section{References}

1. Wernegreen, J. J. Mutualism meltdown in insects: bacteria constrain thermal adaptation. Curr. Opin. Microbiol. 15, 255-262 (2012).

2. Colman, D. R., Toolson, E. C. \& Takacs-Vesbach, C. Do diet and taxonomy influence insect gut bacterial communities? Mol. Ecol. 21, 5124-5137 (2012).

3. Jing, X. et al. The bacterial communities in plant phloem-sap-feeding insects. Mol. Ecol. 23, 1433-1444 (2014).

4. Oliver, K. M., Degnan, P. H., Burke, G. R. \& Moran, N. A. Facultative symbionts in aphids and the horizontal transfer of ecologically important traits. Annu. Rev. Entomol. 55, 247-266 (2010).

5. Buchner, P. Endosymbiosis of Animals with Plant Microorganisms (ed. Buchner, P.) 909 pp. (Interscience, New York, 1965).

6. Douglas, A. Nutritional interactions in insect-microbial symbioses: aphids and their symbiotic bacteria Buchnera. Annu. Rev. Entomol. 43, 17-37 (1998).

7. Degnan, P. H. et al. Dynamics of genome evolution in facultative symbionts of aphids. Environ. Microbiol. 12, 2060-2069 (2010).

8. Ferrari, J., West, J. A., Via, S. \& Godfray, H. C. J. Population genetic structure and secondary symbionts in host-associated populations of the pea aphid complex. Evolution 66, 375-390 (2012).

9. Russell, J. A. et al. Uncovering symbiont-driven genetic diversity across North American pea aphids. Mol. Ecol. 22, 2045-2059 (2013).

10. Oliver, K. M., Moran, N. A. \& Hunter, M. S. Variation in resistance to parasitism in aphids is due to symbionts not host genotype. Proc. Natl. Acad. Sci. USA 102, 12795-12800 (2005).

11. Scarborough, C. L., Ferrari, J. \& Godfray, H. Aphid protected from pathogen by endosymbiont. Science 310, 1781-1781 (2005).

12. Montllor, C. B., Maxmen, A. \& Purcell, A. H. Facultative bacterial endosymbionts benefit pea aphids Acyrthosiphon pisum under heat stress. Ecol. Entomol. 27, 189-195 (2002).

13. Tsuchida, T., Koga, R. \& Fukatsu, T. Host plant specialization governed by facultative symbiont. Science 303, 1989-1989 (2004).

14. Werren, J. H., Baldo, L. \& Clark, M. E. Wolbachia: master manipulators of invertebrate biology. Nat. Rev. Microbiol. 6, 741-751 (2008).

15. Caporaso, J. G. et al. QIIME allows analysis of high-throughput community sequencing data. Nat. Methods 7, 335-336 (2010).

16. Lozupone, C. \& Knight, R. UniFrac: a new phylogenetic method for comparing microbial communities. Appl. Environ. Microbiol. 71, 8228-8235 (2005)

17. Carletto, J. et al. Ecological specialization of the aphid Aphis gossypii Glover on cultivated host plants. Mol. Ecol. 18, 2198-2212 (2009).

18. Augustinos, A. A. et al. Detection and characterization of Wolbachia infections in natural populations of aphids: Is the hidden diversity fully unraveled? Plos One 6, e28695 (2011).

19. Carletto, J., Gueguen, G., Fleury, F. \& Vanlerberghe-Masutti, F. Screening the bacterial endosymbiotic community of sap-feeding insects by terminal-restriction fragment length polymorphism analysis. Entomol. Exp. Appl. 129, 228-234 (2008).

20. Russell, J. A., Latorre, A., Sabater-Munoz, B., Moya, A. \& Moran, N. A. Side-stepping secondary symbionts: widespread horizontal transfer across and beyond the Aphidoidea. Mol. Ecol. 12, 1061-1075 (2003).

21. Desneux, N., Barta, R. J., Hoelmer, K. A., Hopper, K. R. \& Heimpel, G. E. Multifaceted determinants of host specificity in an aphid parasitoid. Oecologia 160, 387-398 (2009).

22. Jones, R. T., Bressan, A., Greenwell, A. M. \& Fierer, N. Bacterial communities of two parthenogenetic aphid species cocolonizing two host plants across the hawaiian islands. Appl. Environ. Microbiol. 77, 8345-8349 (2011).

23. Najar-Rodriguez, A. L., McGraw, E. A., Mensah, R. K., Pittman, G. W. \& Walter, G. H. The microbial flora of Aphis gossypii: Patterns across host plants and geographical space. J. Invertebr. Pathol. 100, 123-126 (2009).

24. Jousselin, E., Cour d'acier, A., Vanlerberghe-Masutti, F. \& Duron, O. Evolution and diversity of Arsenophonus endosymbionts in aphids. Mol. Ecol. 22, 260-270 (2013).

25. Brady, C. M. et al. Worldwide populations of the aphid Aphis craccivora are infected with diverse facultative bacterial symbionts. Microb. Ecol. 67, 195-204 (2014).

26. Duron, O., Wilkes, T. E. \& Hurst, G. D. Interspecific transmission of a male-killing bacterium on an ecological timescale. Ecol. Lett. 13, 1139-1148 (2010)

27. Ferree, P. M., Avery, A., Azpurua, J., Wilkes, T. \& Werren, J. H. A bacterium targets maternally inherited centrosomes to kill males in Nasonia. Curr. Biol. 18, 1409-1414 (2008). 
28. Dale, C., Beeton, M., Harbison, C., Jones, T. \& Pontes, M. Isolation, pure culture, and characterization of “Candidatus Arsenophonus arthropodicus," an intracellular secondary endosymbiont from the hippoboscid louse fly Pseudolynchia canariensis. Appl. Environ. Microbiol. 72, 2997-3004 (2006).

29. Hansen, A., Jeong, G., Paine, T. \& Stouthamer, R. Frequency of secondary symbiont infection in an invasive psyllid relates to parasitism pressure on a geographic scale in California. Appl. Environ. Microbiol. 73, 7531-7535 (2007).

30. Wagner, S. M. et al. Facultative endosymbionts mediate dietary breadth in a polyphagous herbivore. Funct. Ecol. 29, 1402-1410 (2015).

31. Wulff, J. A., Buckman, K. A., Wu, K., Heimpel, G. E. \& White, J. A. The endosymbiont Arsenophonus is widespread in soybean aphid, Aphis glycines, but does not provide protection from parasitoids or a fungal pathogen. Plos One 8, e62145 (2013).

32. Wulff, J. A. \& White, J. A. The endosymbiont Arsenophonus provides a general benefit to soybean aphid (Hemiptera: Aphididae) regardless of host plant resistance (Rag). Environ. Entomol. 44, 574-581 (2015).

33. Turelli, M. \& Hoffmann, A. A. Rapid spread of an inherited incompatibility factor in California Drosophila. Nature 353, 440-442 (1991).

34. Teixeira, L., Ferreira, Á. \& Ashburner, M. The bacterial symbiont Wolbachia induces resistance to RNA viral infections in Drosophila melanogaster. PLoS Biol. 6, 2753-2763 (2008).

35. Hosokawa, T., Koga, R., Kikuchi, Y., Meng, X. Y. \& Fukatsu, T. Wolbachia as a bacteriocyte-associated nutritional mutualist. Proc. Natl. Acad. Sci. USA 107, 769-774 (2010).

36. Liu, S., Chougule, N. P., Vijayendran, D. \& Bonning, B. C. Deep sequencing of the transcriptomes of soybean aphid and associated endosymbionts. Plos One 7, e45161 (2012).

37. Arthofer, W. et al. Hidden Wolbachia diversity in field populations of the European cherry fruit fly, Rhagoletis cerasi (Diptera, Tephritidae). Mol. Ecol. 18, 3816-3830 (2009).

38. Arthofer, W. et al. Allele intersection analysis: a novel tool for multi locus sequence assignment in multiply infected hosts. Plos One 6, e22198 (2011).

39. Kondo, N., Shimada, M. \& Fukatsu, T. Infection density of Wolbachia endosymbiont affected by co-infection and host genotype. Biol. Lett. 1, 488-491 (2005).

40. Vautrin, E. \& Vavre, F. Interactions between vertically transmitted symbionts: cooperation or conflict? Trends. Microbiol. 17, 95-99 (2009).

41. Kondo, N., Nikoh, N., Ijichi, N., Shimada, M. \& Fukatsu, T. Genome fragment of Wolbachia endosymbiont transferred to X chromosome of host insect. Proc. Natl. Acad. Sci. USA 99, 14280-14285 (2002)

42. Hotopp, J. C. D. et al. Widespread lateral gene transfer from intracellular bacteria to multicellular eukaryotes. Science 317, 1753-1756 (2007).

43. Oliver, K. M., Russell, J. A., Moran, N. A. \& Hunter, M. S. Facultative bacterial symbionts in aphids confer resistance to parasitic wasps. Proc. Natl. Acad. Sci. USA 100, 1803-1807 (2003).

44. Oliver, K. M., Moran, N. A. \& Hunter, M. S. Costs and benefits of a superinfection of facultative symbionts in aphids. Proc. R. Soc. Lond., B, Biol. Sci. 273, 1273-1280 (2006).

45. Kikuchi, Y., Hosokawa, T. \& Fukatsu, T. Insect-microbe mutualism without vertical transmission: a stinkbug acquires a beneficial gut symbiont from the environment every generation. Appl. Environ. Microbiol. 73, 4308-4316 (2007).

46. Oliver, K. M., Campos, J., Moran, N. A. \& Hunter, M. S. Population dynamics of defensive symbionts in aphids. Proc. R. Soc. Lond., B, Biol. Sci. 275, 293-299 (2008).

47. Tsuchida, T., Koga, R., Shibao, H., Matsumoto, T. \& Fukatsu, T. Diversity and geographic distribution of secondary endosymbiotic bacteria in natural populations of the pea aphid. Acyrthosiphon pisum. Mol. Ecol. 11, 2123-2135 (2002).

48. Pan, H. et al. Factors affecting population dynamics of maternally transmitted endosymbionts in Bemisia tabaci. PloS One 7, e30760 (2012).

49. Caporaso, J. G. et al. Ultra-high-throughput microbial community analysis on the Illumina HiSeq and MiSeq platforms. ISME J 6, 1621-1624 (2012).

50. Kozich, J. J., Westcott, S. L., Baxter, N. T., Highlander, S. K. \& Schloss, P. D. Development of a dual-index sequencing strategy and curation pipeline for analyzing amplicon sequence data on the MiSeq Illumina sequencing platform. Appl. Environ. Microbiol. 79, 5112-5120(2013).

51. Magoč, T. \& Salzberg, S. L. FLASH: fast length adjustment of short reads to improve genome assemblies. Bioinformatics 27, 2957-2963 (2011).

52. Bokulich, N. A. et al. Quality-filtering vastly improves diversity estimates from Illumina amplicon sequencing. Nat. Methods 10, 57-59 (2013).

53. Edgar, R. C., Haas, B. J., Clemente, J. C., Quince, C. \& Knight, R. UCHIME improves sensitivity and speed of chimera detection. Bioinformatics 27, 2194-2200 (2011).

54. Edgar, R. C. UPARSE: highly accurate OTU sequences from microbial amplicon reads. Nat. Methods 10, 996-998 (2013).

55. DeSantis, T. Z. et al. Greengenes, a chimera-checked 16S rRNA gene database and workbench compatible with ARB. Appl. Environ. Microbiol. 72, 5069-5072 (2006)

56. Wang, Q., Garrity, G. M., Tiedje, J. M. \& Cole, J. R. Naive Bayesian classifier for rapid assignment of rRNA sequences into the new bacterial taxonomy. Appl. Environ. Microbiol. 73, 5261-5267 (2007).

57. Edgar, R. C. MUSCLE: multiple sequence alignment with high accuracy and high throughput. Nucleic Acids Res. 32, 1792-1797 (2004)

\section{Acknowledgements}

We acknowledge Bo Chu, Si-Bao Wang and Li Wang for aphid sample collection. This research was supported by the National Special Transgenic Project of China (2014ZX08011-002).

\section{Author Contributions}

J.C. and S.Z. conceived and designed the experiments. Y.Z. performed the experiments. Y.Z., J.L., C.W. and L.L. analysed the data and prepared the figures and tables. Y.Z. wrote the manuscript. All of the authors reviewed the manuscript.

\section{Additional Information}

Supplementary information accompanies this paper at http://www.nature.com/srep

Competing financial interests: The authors declare no competing financial interests.

How to cite this article: Zhao, Y. et al. Bacterial communities of the cotton aphid Aphis gossypii associated with Bt cotton in northern China. Sci. Rep. 6, 22958; doi: 10.1038/srep22958 (2016) 
(c) (i) This work is licensed under a Creative Commons Attribution 4.0 International License. The images or other third party material in this article are included in the article's Creative Commons license, unless indicated otherwise in the credit line; if the material is not included under the Creative Commons license, users will need to obtain permission from the license holder to reproduce the material. To view a copy of this license, visit http://creativecommons.org/licenses/by/4.0/ 